\title{
DATOS DEL MATERIAL TIPO DE CULICOIDES (DRYMODESMYIA) BAKERI VARGAS (DIPTERA: CERATOPOGONIDAE)
}

\author{
Herón HuerTa \\ Laboratorio de Entomología, InDRE. Carpio 470, Col. Santo Tomás, \\ CP 11340, México D. F. MÉXICO. cerato_2000@yahoo.com
}

RESUMEN

Los adultos macho y hembra de Culicoides bakeri Vargas son redescritos basados en la revisión del material tipo y de otros ejemplares procedentes de la localidad tipo, depositados en la Colección de Artrópodos con Importancia Médica (CAIM).

Palabras Clave: Culicoides bakeri, material tipo, México, Ceratopogonidae.

\section{ABSTRACT}

The adult male and female of Culicoides bakeri Vargas are redescribed based on re-examination of the type material and examination of other specimens from the type locatity, deposited in the Collection of Arthropods of Medical Importance (CAIM).

Keys Words: Culicoides bakeri, type material, Mexico, Ceratopogonidae.

\section{INTRODUCCIÓN}

El subgénero Drymodesmyia Vargas, actualmente cuenta con el registro de 22 especies conocidas al sur de los Estados Unidos de América, de las cuales 14 de ellas tienen una distribución conocida para México (Borkent \& Spinelli, 2000). Particularmente dos especies refieren una distribución restringida para México: Culicoides bakeri Vargas (D.F.) y Culicoides wirthomyia Vargas (Guerrero), ambas conocidas únicamente de la localidad tipo. Diversas especies del género Culicoides Latreille son conocidas por su importancia médica y veterinaria, aunque en contraste muchas de ellas aún son poco conocidas y existen especies pobremente descritas e ilustradas y no se cuenta con claves modernas para separar a todas las especies conocidas del país.

\section{MATERIAL Y MÉTODO}

El objetivo de este trabajo es redescribir a la especie con base en la terminología actual e ilustrar caracteres taxonómicos para reconocer a la especie. Se revisó el material tipo de Culicoides bakeri Vargas (1954) y otro material depositado en la Colección de 
Huerta: Datos de Culicoides bakeri

Artrópodos con Importancia Médica (CAIM) que no pertenece a la serie tipo, el cual fue montado en laminillas con Bálsamo de Canadá siguiendo la técnica descrita por Borkent \& Bissett (1990), la terminología general siguió la propuesta de Downes \& Wirth (1981), Wirth \& Hubert $(1960,1989)$ y para la nomenclatura de las alas se adoptaron las modificaciones indicadas por Spinelli \& Borkent (2004). El material examinado quedó depositado en el acervo de la Colección de Artrópodos con Importancia Médica (CAIM) del Instituto de Diagnóstico y Referencia Epidemiológicos, InDRE (antes Instituto de Salubridad y Enfermedades Tropicales, con siglas ISET, 1939-1989).

\section{RESULTADOS}

\section{Culicoides (Drymodesmyia) bakeri Vargas}

Culicoides bakeri Vargas, 1954. Rev. Inst. Salud. Enf. Trop. 14: 27 (macho holotipo, paratipos macho y hembra; Lomas de Chapultepec, México D. F., figs. ala, palpo, antena, genitales del macho). Wirth \& Hubert. 1960. Ann. Entomol. Soc. Amer., 53:652 (figs. palpo, espermatecas; revisión de las especies como grupo copiosus); Wirth, 1974. Mus. Zool. Univ., Sao Paulo 14:22 (en catálogo de Ceratopogonidae al sur de los Estados Unidos de América); Wirth, Dyce \& Spinelli. 1988. Contrib. Amer. Ent. Inst., 25:8, 24 (Atlas Neotropical del género Culicoides; medidas de la longitud del ala, proporción palpal y costal); Borkent \& Wirth, 1997. Bull. Am. Mus. Nat. Hist., N. Y. 233:62 (en catálogo mundial de la familia Ceratopogonidae); Borkent \& Spinelli. 2000. Contrib. Ent. Internatl., 4:30 (en catálogo de Ceratopogonidae al sur de los Estados Unidos de América).

Redescripción.- Antena con 13 flagelómeros (Fig. 1), los cinco flagelómeros distales más largos que los ocho proximales, estos últimos cortos y anchos; longitud antenal 0.59-0.66mm (0.62mm, $\mathrm{n}=11)$, proporción antenal (AR) 1.00-1.28 (1.10, $\mathrm{n}=11)$, patrón antenal de sensilas celocónicas 1-13 $(n=3)$ (Fig. 1). Ojos moderadamente separados y glabros (Fig. 2); proporción probóscide/cabeza (PHR) 0.93-1.00 (0.95, $n=10$ ); Palpo más largo que la probóscide, longitud $0.21-0.24 \mathrm{~mm}(0.22 \mathrm{~mm}, \mathrm{n}=10)$, tercer segmento palpal ensanchado, con la fosa sensorial profunda (Fig. 2), proporción palpal (PR) 2.00-2.50 (2.29, $\mathrm{n}=3$ ); mandíbula con 20-24 dientes distales finos.

Tórax. Pardo oscuro, con un patrón de líneas longitudinales sobre el mesonoto (Figs. $7,8)$. Patas pardo oscuras, fémures de la pata anterior y media con un anillo claro distal, fémur de la pata posterior pardo oscuro; tibias de todas las patas con anillo claro basal; proporción tarsal de la pata posterior (TR) 2.0-2.2 (2.05, n=10); peine tibial de la pata posterior con 4-5 sedas y el espolón.

Ala con patrón como en la Fig. 9. Segunda celda radial incluida en un área oscura; primera celda radial con la parte distal incluida en un área obscura y la proximal incluida en un área clara; vena $r-m$ incluida en un área clara; celda $r_{3}$ con el área postestigmática clara, área clara adyacente por debajo de la segunda celda radial no confluente con la postestigmática, dos áreas claras redondeadas distales separadas, sin llegar al 
margen distal del ala; base de la vena $\mathrm{M}_{1}$ con un área clara, parte distal de la vena con un área clara muy tenue; celda $\mathrm{m}_{1}$ con un área clara distal redondeada sin alcanzar el margen distal del ala; vena $\mathrm{M}_{2}$ con un área clara basal dispuesta en ambos lados de la vena, parte distal con un área clara muy tenue; celda $\mathrm{m}_{2}$ con un área clara distal redondeada sin llegar al margen distal del ala; vena $\mathrm{CuA}_{1}$ con una pequeña área clara distal muy tenue en el margen del ala; celda cua ${ }_{1}$ con una extensa área clara que no alcanza el margen del ala; vena $\mathrm{CuA}_{2}$ oscura; celda anal con cuatro áreas claras pequeñas y separadas entre sí, las dos áreas anteriores sin alcanzar el margen del ala. Macrosedas abundantes y dispersas en toda el ala; longitud del ala 1.17-1.43mm $(1.33 \mathrm{~mm} ; \mathrm{n}=15)$; anchura de ala $0.58-0.69 \mathrm{~mm}(0.65 \mathrm{~mm}, \mathrm{n}=15)$; proporción costal $(\mathrm{CR})$ 0.48-0.54 (0.51, $\mathrm{n}=15)$. Balancines blancos.

Abdomen. Espermatecas esféricas con cuello corto y delgado (Fig. 3), con las siguientes medidas: $0.042-0.053 \mathrm{~mm}(0.047 \mathrm{~mm}, \mathrm{n}=6) \times 0.030-0.037 \mathrm{~mm}(0.035 \mathrm{~mm}, \mathrm{n}=6)$ y $0.033-0.040 \mathrm{~mm}$ $(0.038 \mathrm{~mm}, \mathrm{n}=6) \times 0.025-0.027(0.026 \mathrm{~mm}, \mathrm{n}=6)$; una tercera espermateca rudimentaria.

Macho. Similar a la hembra, con las habituales diferencias sexuales. Últimos tres flagelómeros distales más oscuros que el resto de los flagelómeros, longitud de la antena $0.61 \mathrm{~mm}(\mathrm{n}=1)$; proporción palpal 1.90-2.3(2.1, $\mathrm{n}=2)$. Patas con el mismo patrón. Ala más estrecha y con las áreas claras más tenues; longitud del ala $1.02-1.10 \mathrm{~mm}$ $(1.06 \mathrm{~mm}, \mathrm{n}=3)$, anchura del ala $0.43-0.47 \mathrm{~mm}(0.45 \mathrm{~mm}, \mathrm{n}=3)$; proporción costal $(\mathrm{CR})$ $0.45-0.47(0.46, n=3)$.

Genitales (Fig. 4). Procesos apicolaterales del noveno terguito largos y delgados, membrana ventral del noveno esternito no espiculada; gonocoxito robusto más largo que gonostilo. Edeago en forma triangular con el ápice estrecho y truncado, apodemas basales cortos y ligeramente doblados (Fig. 5). Parámeros cada uno con un apodema anterolateral corto y robusto, parte media corta y moderadamente ensanchada en la base, parte distal corta y disminuyendo hasta terminar en una punta fina, con una hilera ventral subapical de 4 a 6 espinas cortas y afiladas (Fig. 6).

Material Tipo Examinado: 10". Laminilla. HOLOTIPO. Sin etiqueta de datos de la Localidad, incluye la etiqueta de datos taxonómicos: "Culicoides bakeri", on, 8864, Cet (88012); 19. Laminilla. ALOTIPO. Sin etiqueta de datos de la Localidad, incluye etiqueta de datos taxonómicos: "Culicoides bakeri", 9,96115 , Cet (88009); $20^{\circ} \circ$ " 2 \% $\%$. Laminillas. PARATIPOS. Sin etiquetas de datos de la Localidad, incluye la etiqueta de datos taxonómicos: 1 O". "Culicoides bakeri", 9507, Cet (88011); 1 9. "Culicoides bakeri", 2727, Cet (88010); 10 ". "Culicoides bakeri”, Paratipo, (9178B, lámpara), Lomas, D. F., Cet (88007); 1 9, "Culicoides bakeri”, Paratipo, (Col. A. D. lámpara trampa), Lomas D. F., Cet (88008); (laminilla rota). Alfiler entomológico: "bakeri", Paratipo, 9466, 9, Lomas (escrito con tinta negra); 9 , Paratipo, con los mismos datos anteriores. Ejemplar completo, adherido a la pinereta con goma entomológica (Figs. 7, 8).

Otro material examinado (laminillas):10", México D. F. Lomas de Chapultepec, 15-ago1939, Lámpara Trampa, M. F. 8755B, Col. A. Dampf; 9 \$ 9 , mismo datos, abril-1939, M. F. 8468A; 2 ○Q, mismo datos, 13-jun-1939, M. F. 8522A; 2 Q 9 , mismos datos, 20-sept.1939, M. F. 8813I. 2 9 9,2 ơ O', mismos datos, 23-ago-1941, M. F. 9530. 


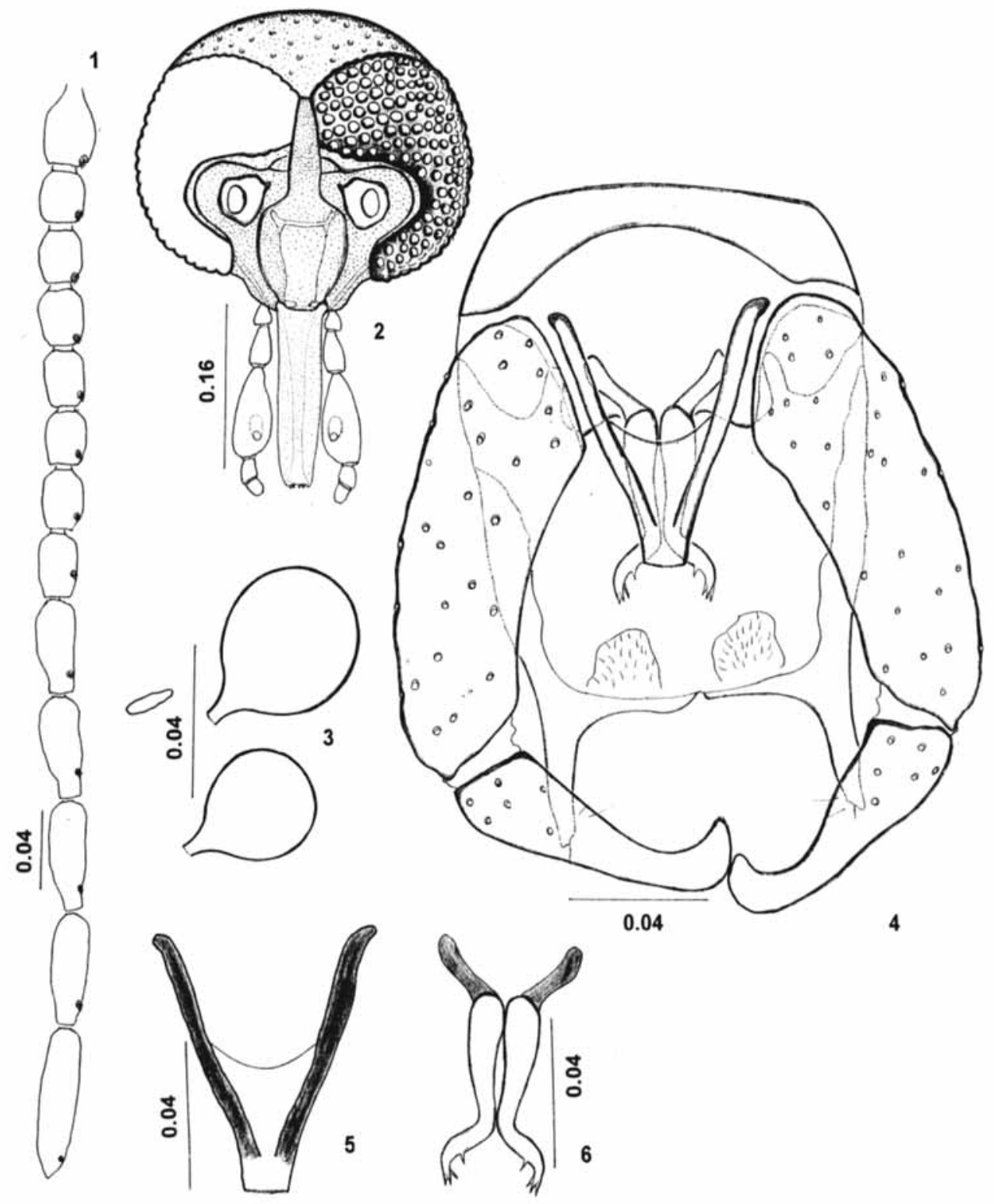

Figuras 1-6

Culicoides bakeri Vargas. Hembra: 1-3; Macho: 4-6. 1. Antena; 2. Cabeza (vista dorsal); 3. Espermatecas; 4. Genitales (vista ventral); 5. Edeago (vista ventral); 6. Parámeros (vista ventral). 

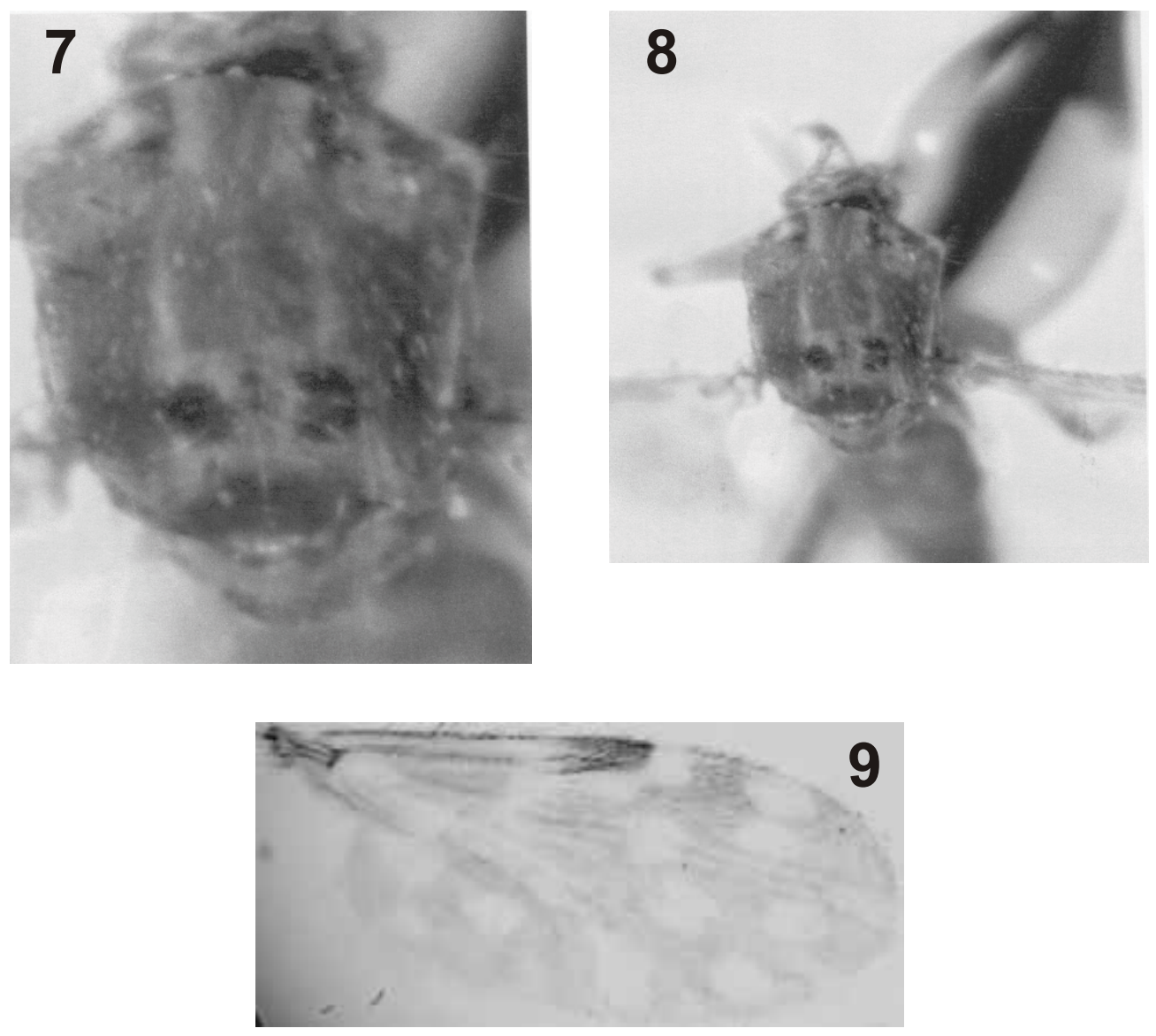

Figuras 7-9

Culicoides bakeri Vargas. Hembra. 7. Patrón de coloración del tórax (vista dorsal); 8. Tórax en vista dorsal, paratipo montado en alfiler entomológico; 9. Ala (vista dorsal).

\section{DISCUSIÓN}

Wirth \& Hubert (1960) en la revisión del grupo copiosus, examinaron paratipos de Culicoides bakeri Vargas, complementando la descripción de algunos caracteres de la hembra y una detallada descripción de los genitales del macho. Esta especie puede reconocerse fácilmente de otras especies del subgénero Drymodesmyia Vargas por la forma de los parámeros del macho y en el caso de la hembra por la forma del frontoclípeo, espermatecas, patrón del tórax y ala.

Actualmente la localidad tipo (Lomas de Chapultepec), se encuentra embebida en un área urbanizada hacia el noroeste de la Ciudad de México, Distrito Federal. El área 
Huerta: Datos de Culicoides bakeri

natural más cercana a la localidad tipo, se conoce como Bosque de Chapultepec donde se han realizado dos intentos ocasionales de colecta y no se pudieron obtener ejemplares de esta especie.

\section{LITERATURA CITADA}

Borkent A. \& B. Bissett. 1990. A revision of the Holarctic species of Serromyia Meigen (Diptera: Ceratopogonidae). Sys. Entomol. 15: 153-217.

Borkent A. \& G. R. Spinelli. 2000. Catalog of the new world biting midges south of the United States of America (Diptera: Ceratopogonidae). Contrib. Ent. Internatl. 4(1): 1-107.

Borkent A. \& W. W. Wirth. 1997. World species of biting midges (Diptera: Ceratopogonidae). Bull. Am. Mus. Nat. Hist., N.Y. 233:1-257.

Downes J. A. \& W. W. Wirth. 1981. Ceratopogonidae, Chapter 28. Pp. 393-421 In: J. F. McAlpine, B. V. Peterson, G. E. Shewell, H. J. Teskey, J. R. Vockeroth \& D. M. Wood (eds.). Manual of Nearctic Diptera, Vol. 1. Agriculture Canada Research Branch, Monograph No. $27,674 \mathrm{pp}$.

Spinelli G. R. \& A. Borkent. 2004. New species of Central American Culicoides Latreille (Diptera, Ceratopogonidae) with a synopsis of species from Costa Rica. Proc. Entomol. Soc. Wash., 106(2):361-395.

Vargas L. 1953. Culicoides wirthomyia n. sp. y Culicoides stigmalis Wirth, 1952 (Insecta, Diptera). Rev. Inst. Salub. Enfer. Trop. 13:227-233.

- 1954. Dos nuevas especies de Culicoides Mexicanos (Diptera, Heleidae). Rev. Inst. Salub. Enfer. Trop. 14(1):25-32.

Wirth W. W. 1974. Family Ceratopogonidae. 14:1-89 In: N. Papavero (ed). A catalog of the Diptera of the Americas south of the United States, Mus. Zool., Univ. Sao Paulo, Fasc.14.

Wirth W. W., A. L. Dyce \& G. R. Spinelli. 1988. An atlas of wings photographs, with a summary of the numerical characters of the Neotropical species of Culicoides (Diptera: Ceratopogonidae). Contrib. Amer. Ent. Inst., 25(1):1-72.

Wirth W. W. \& A. A. Hubert. 1960. Ceratopogonidae (Diptera) reared from cacti, with a review of the copiosus group of Culicoides. Ann. Entomol. Soc. Am. 53:639-658.

1989. The Culicoides of Southeast Asia (Diptera: Ceratopogonidae). Mem. Amer. Ent. Inst. 44, $508 \mathrm{pp}$.

Recibido: 24 de julio 2006

Aceptado: 18 de octubre 2006 